
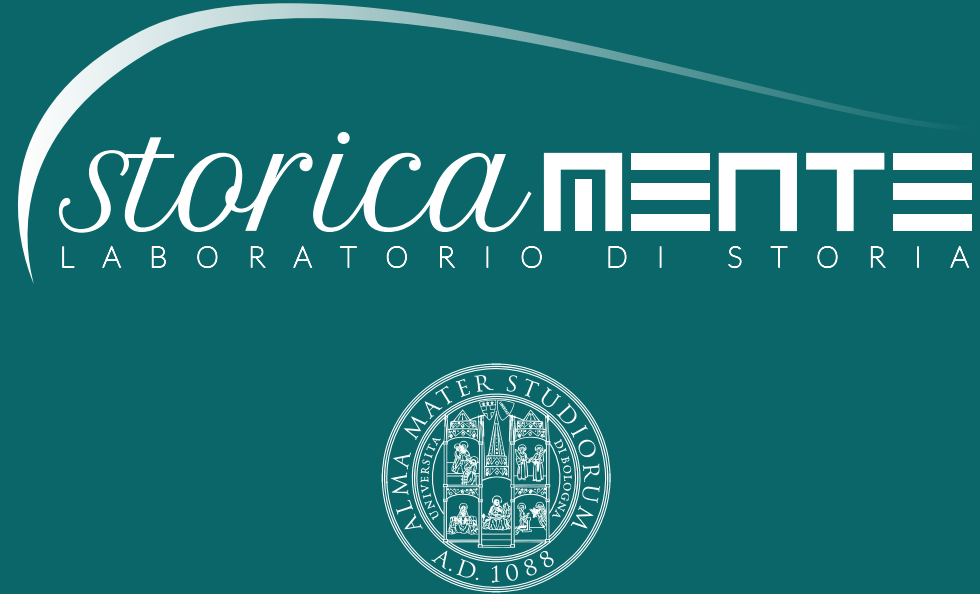

ALMA MATER STUDIORUM

Università di Bologna

Dipartimento di Storia Culture Civiltà

COMUNICARE STORIA

LA STORIA A SCUOLA OGGI.

INSEGNARE STORIA

NELLA SCUOLA PRIMARIA 


\section{STORICAMENTE.ORG}

\section{Laboratorio di Storia}

Marco Tibaldini

Giochi didattici e storia nella scuola primaria

Numero 15 - 2019

ISSN: 1825-411X

Art. 51

pp. 1-22

DOI: $10.12977 /$ stor797

Editore: BraDypUS

Data di pubblicazione: 05/10/2020

Sezione: Comunicare storia: "La Storia a scuola oggi. Insegnare storia nella scuola primaria” 


\title{
Giochi didattici e storia nella scuola primaria
}

\author{
MARCO TIBALDINI \\ Libera Università di Bolzano, Facoltà di Scienze della \\ Formazione
}

The history course at primary school could be extremely challenging for both, teachers and pupils: the understanding of history imply the development of a certain mindset and to learn a huge amount of information. Shifting the educational perspective from data learning to cognitive operations could help teachers to find more interactive and effective methodologies of teaching, transforming the 'history course' into an 'history lab'. In particular, among the cognitive operations, 'theming' is the most intriguing and stimulating to teach and learn. Games and board games expressly designed for history classes could help to make it also funny.

\section{Una didattica ludica per la storia}

Tra le innovazioni metodologiche introdotte nella didattica della storia durante gli ultimi decenni, risulta molto interessante la scelta di Antonio Brusa di includere l'attività ludica nel curricolo di storia per la scuola primaria e secondaria. La sua idea non era quella di rendere gradevole il contenuto del manuale, con cruciverba e labirinti vari, o semplicemente di migliorare l'approccio dell'alunno nei confronti della disciplina, ma di scendere in profondità nella prassi didattica: «l'errore principale di questi giochiziari è che nascono in un ambiente pedagogico-editoriale nel 
quale si teorizza che la "forma" [...] abbia il potere magico di rendere fruibile, gradevole, motivante e via energizzando, qualsiasi "sostanza" [...] non è così. Un gioco storico ha la possibilità di appassionare [...] se nasce da un problema storico attraente. Da una storia viva.» (Brusa 2012, 17-8). Insieme al suo gruppo di ricerca Historia Ludens si è dedicato alla progettazione di giochi appositamente pensati per il contesto scolastico. Queste attività tengono in considerazione la durata di una lezione di storia, ma anche le caratteristiche peculiari del contesto classe, intesa come gruppo di studenti e spazio/ambiente/aula. Si adattano perfettamente alle necessità del docente, fornendo dei materiali stampabili, pronti all'uso e adeguati alla scansione tematica del curricolo di storia. L'esperienza sviluppata da Brusa, unitamente agli studi sull'epistemologia disciplinare e sulla metodologia didattica condotti da Ivo Mattozzi, hanno portato alla realizzazione del progetto Giochi \& Civiltà: un percorso ludicodidattico per la scuola primaria che, con 5 attività annuali, segue lo sviluppo cognitivo del bambino e la progressione del curricolo di storia per le classi III-IV-V (giochiecivilta.jimdofree.com/giochi). Le attività sono state pensate per corrispondere alle finalità della didattica disciplinare, sia sotto il profilo contenutistico che metodologico. L'alunno si trova così a lavorare parallelamente sul piano delle conoscenze e quello

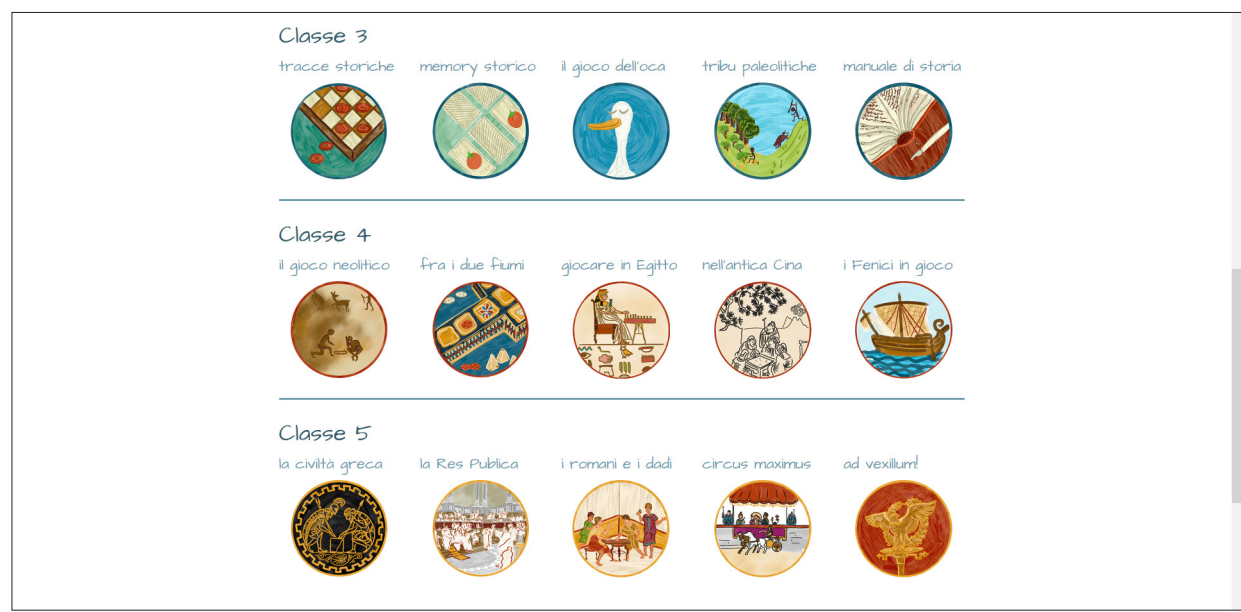

Le attività del progetto Giochi \& Civiltà con cinque giochi didattici di storia per le classi terza, quarta e quinta della scuola primaria (https://giochiecivilta.jimdofree.com/giochi). 
delle competenze, gioca con le tracce storiche e produce informazioni a partire da esse applicando quelle particolari procedure cognitive che servono per costruire una conoscenza storica.

Ogni lezione inclusa nel progetto 'Giochi \& Civiltà' prende le mosse da un'attività di gioco libero, semplice e divertente, nella quale i bambini, spinti dall'entusiasmo e dalla competizione, arrivano a compiere delle elaborazioni cognitive complesse come l'analisi di tracce storiche autentiche, l'individuazione dei collegamenti fra presente e passato, individuo e società, scala geografica locale e globale, fatti storici e processi di trasformazione.

Il gioco contribuisce poi a creare un contesto d'apprendimento cooperativo che coinvolge gli alunni sul piano emotivo, cognitivo e anche sociale. Sotto il profilo contenutistico si cerca di favorire la strutturazione dei 'concetti fondanti' propri della disciplina storica come quelli di 'società', 'ambiente', 'tracce storiche' (Coltri 2019), mentre sotto il profilo cognitivo si intende sollecitare l'utilizzo delle competenze storiche di base: 'tematizzazione', 'spazializzazione', 'temporalizzazione', 'problematizzazione' (Mattozzi 1990a, 76-83; 1990b, 15, 21-33; Perillo 2011). La progettazione di ciascuna attività poggia infatti su di un'analisi delle conoscenze fondamentali per la comprensione di un determinato periodo storico, e sul modo in cui queste competenze storiche ci richiedono di ragionare. In questo articolo si espone quindi un esempio di attività ludico-didattica del progetto 'Giochi \& Civiltà', strutturata per veicolare delle specifiche conoscenze e per mettere all'opera una delle competenze fondamentali, quella della tematizzazione.

\section{Che cos'è la tematizzazione: fra epistemologia e didattica}

Nell'attività dello storico professionista «tematizzare significa circoscrivere, delimitare, escludere. Rinunciare alla pretesa di un'osservazione che vorrebbe essere totale, e assumere invece un determinato punto di vista al quale 
subordinare la ricerca e rispetto al quale selezionare e rendere pertinenti le osservazioni» (Mattozzi 1990a, 76). Quando si tematizza si esplicita il tema di una ricerca e se ne circoscrivono gli estremi temporali e spaziali. "Tematizzare vuol dire individuare un argomento in un tempo e in uno spazio definiti: la tematizzazione è un elemento, un criterio di discriminazione tra informazioni pertinenti con il tema della ricerca e informazioni che non sono pertinenti, tra aspetti del passato che vanno indagati e che non vanno indagati» (Mattozzi e Zerbini 2006, 27).

La tematizzazione è una fase fondamentale che lo storico deve attraversare per svolgere la propria attività di ricerca e dare risposta ai suoi bisogni conoscitivi: è una fase in cui si mette a fuoco il tema dello studio, come ad esempio 'l'alimentazione nella Roma imperiale', poi si cercano le tracce storiche che possono esservi riferite, e infine si selezionano in esse solo quegli aspetti che sono funzionali allo studio di questo tema. Proseguendo con l'esempio della cucina romana: un antico cucchiaio di stagno ci potrebbe dare informazioni anche a riguardo della metallurgia in età romana, ma in riferimento al nostro tema questo approfondimento sarebbe inutile. L'attività di tematizzazione riveste un ruolo fondamentale anche quando lo storico veicola le proprie conoscenze attraverso un testo storiografico, fungendo da criterio ordinatore, facendo sì che non si crei confusione fra temi e sottotemi e che il testo trasmetta i contenuti in modo chiaro ed efficace. Perché questo processo di trasmissione di una nuova conoscenza si concretizzi è tuttavia necessario che il destinatario del testo storiografico, oltre a possedere già un proprio sistema di conoscenze al quale collegare quelle contenute nel testo, sia a sua volta in grado di tematizzarla. Se così non fosse non sarebbe in grado di seguire la tematizzazione proposta dall'autore, di farla propria ed acquisirla, oppure di reagire ad essa, di creare nella sua mente delle nuove macro-aree tematiche, di perfezionare quelle già esistenti, di stabilire fra loro delle nuove connessioni. Per far ciò dal punto di vista cognitivo dovrebbe quindi disporre di: 
- una memoria con un sufficiente numero di punti d'accesso, di nodi ai quali collegare le nuove informazioni;

- un sistema di riconoscimento delle informazioni flessibile e che sappia riconoscere ed organizzare rapidamente il materiale informativo in ingresso.

Queste esigenze del nostro sistema cognitivo, di accessibilità e rapidità, lo portano a compiere spesso delle scelte di tipo 'euristico', che cercano di risolvere un problema attraverso un procedimento più intuitivo che razionale, più disordinato che metodologico, e perciò fa ricorso a quelle categorie mentali e verbali al momento più agevoli, ma che potrebbero non essere quelle più adeguate (ad esempio quando i bambini a scuola ci danno delle definizioni imprecise usando parole generiche come 'è un coso - è una cosa cosa', ma che al momento sono quelle più a portata di mano). Prima di acquisire delle informazioni quindi bisognerebbe sviluppare quelle categorie concettuali ottimali e funzionali alla loro comprensione. Questi contenitori concettuali aiutano infatti ad individuare dei tratti di similarità tra le informazioni che vi vengono inserite ed agevolano la creazione di conoscenze e sistemi di conoscenze. Spesso tali categorie sono però dei cantieri aperti, sono dei confini flessibili che talvolta si modificano a seconda delle informazioni con le quali veniamo in contatto e che pur non rientrando completamente nella nostra griglia di catalogazione mostrano dei chiari punti di collegamento con una o più aree concettuali. In questo caso siamo costretti a ridisegnare la nostra griglia, ad inventare nuove definizioni per le sue categorie, a rifinirle a livello verbale.

Pensiamo ad esempio a come si svolge la didattica dei 'Quadri di Civiltà'. Questa si realizza attraverso l'analisi ricorsiva di alcuni 'indicatori' che fungono da lenti di ingrandimento e attraverso i quali osserviamo le civiltà antiche (come 'società', 'tecnologia', 'economia'...), possiamo notare che si tratta essenzialmente di un'attività di rielaborazione tematica pensata per portare i bambini a configurare le 'informazioni' in 'conoscenze' attraverso dei processi di tematizzazione. I manuali pre- 
sentano diverse informazioni in modo scollegato e puntuale che, se non vengono rielaborate dai bambini, se non vengono collegate fra loro su base tematica in un quadro unitario che abbia un senso esplicativo, restano lettera morta e vengono dimenticate in breve tempo. La didattica dei 'Quadri di civiltà' propone di far analizzare queste informazioni agli alunni e di raggruppare in macro-categorie che raccolgono tutte quelle riferite allo stesso tema. Ad esempio, delle asserzioni come:

- i sumeri inventarono la ruota e la vela;

- i sumeri erano abili commercianti;

- gli artigiani sumeri producevano gioielli con oro e pietre preziose importate dall'India;

Sono tutte informazioni singole e scollegate fra loro, che però possiamo raccogliere all'interno di un'unica grande area tematica che chiamiamo 'Economia'. Collegandole fra loro possiamo iniziare a costruire un quadro esplicativo dell'economia sumera, dell'ampiezza della loro azione commerciale e della loro centralità economica su scala regionale. Questo diventerà poi a sua volta un sotto-tema del più ampio 'Quadro di Civiltà', che raggruppa tutte le sotto-tematiche riferite ai sumeri.

La tematizzazione che viene proposta agli alunni della scuola primaria dai 'Quadri di Civiltà' è quindi pensata per aiutarli a formare nella propria mente delle categorie interpretative della realtà, che poi risultano applicabili sia ad ogni epoca passata che al loro presente. Questi criteri dovrebbero aiutarli ad organizzare le informazioni che incontrano in un testo, in un documentario televisivo, in un fumetto o in una gita scolastica. Imparando ad organizzare e riordinare le informazioni a seconda di queste categorie interpretative sviluppano anche la capacità di individuare fra di esse delle connessioni, andando così a strutturare delle conoscenze.

Lo storico professionista restringe il campo della sua ricerca attraverso un'attività di tematizzazione che orienta la sua attenzione verso alcune specifiche tracce storiche a discapito di altre, e lo porta a rilevare per ciascuna di esse solo quelle informazioni che sono funzionali al tema. 
Nello stesso modo anche gli alunni quando realizzano dei 'Quadri di Civiltà' selezionano, catalogano e riorganizzano le informazioni.

\section{I manuali e i sussidiari di storia}

Sfortunatamente le cose non sempre vanno così. Anche se l'editoria scolastica ha recepito l'importanza della didattica per 'Quadri di Civiltà', ne ha frainteso il significato: in origine questo approccio didattico era stato pensato come un'attività pratica da svolgere insieme agli alun$\mathrm{ni}$, come una riformulazione delle informazioni presentate dal manuale secondo delle tematiche individuate dai bambini, mentre i sussidiari tendono a fornire dei grafici spazio-temporali e degli schemi dei 'Quadri di Civiltà' già fatti e già affollati da scritte, colori e immagini, riducendo così la possibilità di manipolarli e modificarli insieme ai bambini. Il problema maggiore della didattica della storia nella scuola primaria è proprio questo: al contrario di altre discipline, in ambito scolastico non si insiste sulla formazione delle competenze storiche e sullo sviluppo di conoscenze, ma si tende a focalizzarsi invece sulla trasmissione di informazioni puntuali. L'idea generalizzata è che la formazione storica sia destinata alla formazione di un 'sapere' anziché di un 'saper fare'.

Le competenze storiche di base, fra le quali anche la tematizzazione, non vengono così presentate dai manuali (ciascuno può controllare sul proprio manuale e sussidiario), e generalmente neanche nelle guide per docenti, e così nemmeno sollecitate attraverso degli esercizi e delle attività pratiche. Ad aggravare la situazione c'è il fatto che i manuali, soprattutto quelli della scuola primaria, presentano una selezione di informazioni assai scomoda per il docente e si concentrano più sui tratti folkloristici delle antiche civiltà piuttosto che su quegli elementi che potrebbero aiutarci a sviluppare delle analisi trasversali fra di esse, che potrebbero anche consentirci di collegare quelle epoche lontane con la nostra. Ad esempio, quelli che nella 'Didattica dei Quadri di 
Civiltà' vengono individuati come 'indicatori', come categorie attraverso le quali analizzare le civiltà, non vengono trattati equamente in tutti i capitoli del manuale: dei sumeri viene presentato solo l'aspetto economico e tecnologico, degli egizi quello agricolo e religioso, degli assiri l'aspetto militare e dei babilonesi quello legislativo. Non è facile trovare nei sussidiari dei punti di contatto fra le varie civiltà, le cronologie vengono spezzate, le mappe hanno delle prospettive geografiche così ristrette che i bambini non riescono ad accostarle nella loro mente ed a ricomporre un quadro generale (anche in questo caso, possiamo trovare riscontro di ciò sui manuali che abbiamo in adozione per l'anno corrente).

Infine, $i$ testi dei sussidiari sono estremamente brevi, riassumono in una riga tematiche complesse e danno per scontate diverse conoscenze preliminari (dire ad esempio che gli egizi erano degli ottimi agricoltori, implica che i bambini sappiano come si pratica l'agricoltura e cosa significhi farlo in modo 'ottimale'), e sono accostati ad immagini spesso poco significative. Tutti questi fattori ostacolano le possibilità dei bambini di mettere all'opera delle competenze storiche, di riorganizzare le informazioni che trovano nel testo, di ritematizzarle, ed in generale di svolgere su di esse delle attività cognitive come il pensiero critico e la soluzione di problemi, diversamente dalla componente di tipo mnemonico.

Questo a mio avviso è un primo nodo cruciale relativo alla tematizzazione nell'ambito della didattica della storia: generalmente a scuola i bambini non si esercitano nella tematizzazione, non creano delle proprie tematizzazioni, ma subiscono quelle altrui che non consentono loro altre possibilità se non quella dell'apprendimento mnemonico. Non raffinando quei procedimenti mentali che portano alla definizione di un tema fanno poi molta più fatica ad assimilare le informazioni e configurarle in conoscenze attraverso un percorso di 'personalizzazione', che consenta loro di sentire quelle conoscenze come personali e strutturali per la propria visione del mondo. 


\section{La tematizzazione come esito di un processo mentale}

Tematizzare significa selezionare, definire ed esplicitare un tema, ma arrivare a questa selezione, definizione ed esplicitazione è già un enorme sfida cognitiva!

Questo accade perché la tematizzazione non è solo un criterio ordinatore e il punto di partenza di una ricerca storica, ma è anche il punto di arrivo di una serie di pregresse attività mentali, di una precedente attività che alternando operazioni cognitive e costruzioni concettuali porta alla strutturazione di un tema. Scegliere di studiare ad esempio 'l'alimentazione romana in epoca imperiale' richiede che a monte ci siano una curiosità, delle conoscenze e delle concettualizzazioni pregresse. Imparare a tematizzare non significa quindi soltanto sapersi attenere a un criterio selezionatore, ma innanzitutto saperlo stabilire, compiendo in modo efficace tutte le procedure che ci portano a metterlo a fuoco nella nostra mente.

A livello cognitivo, gli stessi meccanismi che utilizziamo per cercare delle tracce storiche pertinenti a un tema, le utilizziamo anche per formulare il tema stesso. In entrambi i casi sviluppiamo delle associazioni mentali fra concetti che ci sembrano pertinenti, coerenti o correlati l'uno all'altro, solo che in un caso partiamo da un tema di riferimento, mentre nell'altro lo dobbiamo creare ex novo. Facciamo un esempio: oggi noi studiamo la civiltà minoica, è un tema presente in tutti i manuali e sussidiari. Quando l'archeologo britannico Arthur Evans condusse le sue campagne di scavo sull'isola di Creta, inaspettatamente rinvenne tracce di epoche così diverse da non saper come interpretarle, come definirle. Quei reperti sfidavano le categorie concettuali con le quali Evans guardava la storia e perciò faticava a tematizzarle, a definire un tema che potesse racchiuderle tutte in modo organico, che le identificasse. In seguito, arrivò a stabilire delle periodizzazioni per la storia cretese e a definire i tratti di una civiltà che si era sviluppata sull'isola fra il 2700 e il 1200 a.C., e per la quale, non avendo un nome, ne inventò uno di fantasia. La chiamò 'civiltà minoica' in onore della figura 
mitologica del re Minosse. Solo al termine di questa 'messa a fuoco' del tema poté calarsi nei panni dello storico, selezionare le tracce che gli sembravano più rappresentative e iconiche, ricostruire fatti storici ed eventi relativi a questa civiltà, e comunicare la propria interpretazione attraverso un testo che poi divenne uno dei grandi classici della letteratura archeologica. 'Grazie a questa riflessione di Evans oggi noi possiamo tematizzare della civiltà minoica nelle nostre lezioni di storia, quindi trattare un tema che, prima di venir così formulato, non era preso in considerazione, non era identificabile.

Fin dai primi mesi di vita impariamo a stabilire delle associazioni tra le tracce mnemoniche dei vari fenomeni di cui facciamo esperienza e che impariamo a categorizzare su base spaziale, temporale, di soggetto e d'azione. Le cose accadono in un luogo, in un tempo, con un soggetto che compie o subisce un'azione, e rielaboriamo ogni nostra esperienza sensoriale o intellettuale identificando in essa queste quattro componenti. In questo modo trasformiamo una semplice 'memoria' in un 'ricordo' di tipo episodico. Il bambino inizia ad associare tra loro i ricordi di fatti vissuti in prima persona che presentano delle similarità di tipo spaziale perché accadono sempre nello stesso posto: questo lo porta ad organizzare quindi le sue memorie su base spaziale, ma anche a concettualizzare gli spazi domestici in cui vive. Successivamente inizia ad associare tra loro i ricordi su base temporale, concettualizzando la dimensione temporale dell'esistenza e sviluppando l'idea di un prima ed un poi, di un passato, un presente e un futuro. Due altre tipologie di pertinenze fondamentali sono quella su base 'soggettiva', ossia che categorizza le esperienze in base alla persona o all'oggetto coinvolto, e di 'azione', che associa quelle esperienze caratterizzate dallo svolgimento della stessa attività. Attraverso questa continua rielaborazione dei propri ricordi e alla loro catalogazione si arriva allo sviluppo delle prime astrazioni simboliche, concettualizzazioni e costruzioni tematiche, e 
alla stratificazione di alcuni schemi operativi mentali che ci aiutano a rapportarci con il passato.

\section{La fase della personalizzazione}

Sviluppare una 'competenza nella tematizzazione' non significa sapersi attenere il più rigidamente possibile a una norma catalogatrice, ma imparare a stabilire di volta in volta quella norma catalogatrice che ci serve per comprendere una parte della realtà verso la quale abbiamo degli interessi. L'origine della volontà di tematizzare si trova nella curiosità personale e prende forma attraverso la rilevazione di alcune similarità tra $\mathrm{i}$ fenomeni di cui facciamo esperienza sensoriale e intellettuale. Se queste esperienze corrispondono a delle nostre necessità e intenzioni cognitive, la nostra fantasia le amplifica e, realizzandone un'immagine mentale, le esplora, aumentando ancora di più la nostra curiosità personale, fondamentale per innescare un processo cognitivo (Tibaldini e Scacchi 2017). La fantasia è quanto di più originale ci sia in noi: ciò che passa attraverso di essa viene 'personalizzato' e assume dei caratteri di 'personalità' per poi essere innestato all'interno delle nostre strutture concettuali e dei nostri sistemi di conoscenze, contribuendo ad evolverli. Quando mettiamo a fuoco un tema, quando lo sagomiamo nella nostra mente, ancor prima di iniziare a fare delle ricerche al riguardo, lo sentiamo parte di noi, del nostro modo di vedere il mondo, e pregustiamo il piacere che la sua esplorazione ci darebbe. Per venire acquisita, una nuova conoscenza deve necessariamente essere personalizzata dal discente, anche attraverso la fantasia.

Per poter costruire una conoscenza in forma personale, per poter quindi 'personalizzare' quelle conoscenze che il manuale intende trasmettere e per integrarle con un patrimonio di conoscenze pregresse, è necessario che $\mathrm{i}$ bambini imparino a praticare la tematizzazione in modo attivo, non a subirla. Proviamo a pensare cosa succederebbe se togliessimo tut- 
ti i titoli ai vari capitoli del sussidiario e chiedessimo ai bambini di darne di propri al termine della lettura: imparerebbero a tematizzare in modo molto efficace e sedimenterebbero in modo molto più stabile e duraturo le conoscenze acquisite attraverso un procedimento di apprendimento attivo e personalizzato. Darebbero magari dei titoli improbabili ai vari capitoli, ma sarebbero dei titoli che sentirebbero propri, sentirebbero di aver definito da sé i contorni di un tema di ricerca. Oppure, basterebbe soltanto che nei manuali scolastici si motivassero le tematizzazioni specificando perché vengono identificati quegli specifici argomenti e come vengono stabiliti i loro estremi spazio-temporali: sarebbe già un modo per esplicitare le riflessioni sulle quali si è fondata la tematizzazione e consentire anche gli alunni di ripercorrerle mentalmente. Oltre a ciò, resta però il fatto che la didattica della storia nella scuola primaria ha bisogno di ben altri materiali, che possano trasformare un paradigma didattico di tipo trasmissivo in uno operativo e laboratoriale.

\section{Un laboratorio di storia fatto con i giochi}

Volendo dotare i docenti di uno strumento in grado di sollecitare le competenze storiche degli alunni, di portarli a riformulare le conoscenze su base personale, di suscitare il loro interesse nei confronti della disciplina, si è deciso di rinnovare la proposta di una didattica ludica per il curricolo di storia nella scuola primaria. Attraverso essa si intende sollecitare i bambini in diversi modi e a diversi livelli: dal punto di vista psicologico incuriosendoli e coinvolgendoli emotivamente, ed al contempo sfidare le loro competenze cognitive, spingendoli così a compiere percorsi conoscitivi complessi composti da più passaggi inferenziali. In riferimento all'epistemologia disciplinare si intende portare gli alunni ad applicare in modo ricorsivo le operazioni di analisi delle tracce storiche, affinché imparino il metodo dell'indagine storica. Come materiale di gioco si utilizzano le informazioni presenti nel manuale, 
dove però il vincitore non è colui che sa memorizzarle, ma chi riesce a collegarle e utilizzarle in modo funzionale. In riferimento agli attuali orientamenti pedagogici, l'attività viene svolta in gruppo e la situazione di cooperative learning che si viene a creare distribuisce il carico di lavoro intellettuale e il peso emotivo della prestazione fra i vari membri del gruppo, che non si sentono intimiditi dalla difficoltà del lavoro o messi sotto verifica, ma motivati dalla sfida. Sotto il profilo sociale essa comporta una collaborazione fra individui con approcci e metodi operativi differenti che mettono a confronto diverse prospettive, idee, suggestioni, percezioni, categorizzazioni e strutture mentali. Inoltre, l'attività ludica proposta rende partecipi i bambini, consentendo loro di applicare le proprie strategie cognitive e di personalizzare la situazione formativa, che si configura come un'intensa occasione di apprendimento esperienziale 'per scoperta'.

\section{Memory Storico: un gioco per imparare a tematizzare}

Il Memory storico è una delle attività incluse nel percorso 'Giochi e Civiltà' per la classe terza ed è completamente dedicata al potenziamento di quelle competenze che stanno alla base della tematizzazione. L'esperienza inizia con il setting dello spazio didattico creando 4 isole di lavoro e dividendo $\mathrm{i}$ bambini in 4 gruppi, bilanciati fra loro dal punto di vista sociale, intellettuale e agonistico. A ciascuno di questi vengono distribuiti 6 fogli con due identici set di 24 carte ciascuno. I bambini le ritagliano, le dispongono capovolte sul banco e giocano al Memory con queste carte per 30-40 minuti senza alcun intervento da parte del docente. Questa fase di gioco libero ha lo scopo di riattivare cognitivamente i bambini, di farli tornare ad essere attivi, di interessarli e farli sentire partecipi della lezione. Possono alzarsi in piedi, stare in ginocchio sulla sedia, parlare ad alta voce, muoversi attorno al tavolo. È un momento di svago ricreativo e perciò anche di riappropriazio- 
ne di sé. Durante la fase di gioco libero però poniamo anche le basi per un successivo lavoro, che sarà cognitivamente molto più intenso e che costituirà il nucleo formativo della lezione. Mentre giocano al Memory i bambini familiarizzano con delle informazioni storiche: sulle carte sono infatti raffigurati mammut, dinosauri e fossili marini. Anche sotto il profilo cognitivo utilizziamo questo approccio graduale: nel gioco del Memory vince il giocatore che riesce a ritrovare più coppie possibili, così i bambini si sentono psicologicamente gratificati quando ricompongono una coppia, e rivolgono la loro attenzione ai tratti di similarità ed identità fra le carte. Si iniziano a orientare la loro attenzione e la loro percezione verso l'associazione di ciò che è simile e identico, quindi verso quei procedimenti mentali che sono alla base della tematizzazione.

Terminata questa fase del gioco il docente spiega che le tessere usate per giocare al Memory nascondono al loro interno 6 sequenze, o meglio "6 storie" composte da 4 carte ciascuna e si chiede ai gruppi di ricomporle. Quando i membri di un gruppo credono di aver ricomposto una sequenza alzano la mano e il docente, dopo averla osservata, stabilisce se è corretta o meno. Non può dare alcuna indicazione, ma specificare solamente quante carte sono giuste e quante sbagliate senza indicarle. I bambini sapranno di aver ricomposto una sequenza per metà, con due carte corrette su un totale di 4, oppure di averla composta nel modo giusto, ma di non aver messo le carte nel corretto ordine logico/cronologico. Come potete immaginare, questa fase della lezione viene facilmente interpretata dai bambini come un gioco a squadre, un fatto che li porta a intensificare il loro coinvolgimento emotivo, e di conseguenza anche intellettuale.

Questo compito richiede ai bambini di analizzare le carte e identificare le diverse sequenze, procedendo per tentativi e ipotesi che sono inconsapevolmente fondate su dei continui processi di tematizzazione: osservando una carta cercano di ipotizzarne un tema di riferimento e cercano altre carte ad esso riferibili, arrivando spesso a intuire di averlo 
formulato in modo impreciso, oppure di aver seguito le tracce sbagliate. I bambini capiscono quindi da soli di aver commesso un errore, si auto correggono, abbozzano dei nuovi temi e riprendono con la ricerca. Questa ricorsività, partendo da una condizione di padronanza completa, porta i bambini a confrontarsi con delle sfide sempre più ardite e a effettuare delle associazioni concettuali sempre più complesse. Si

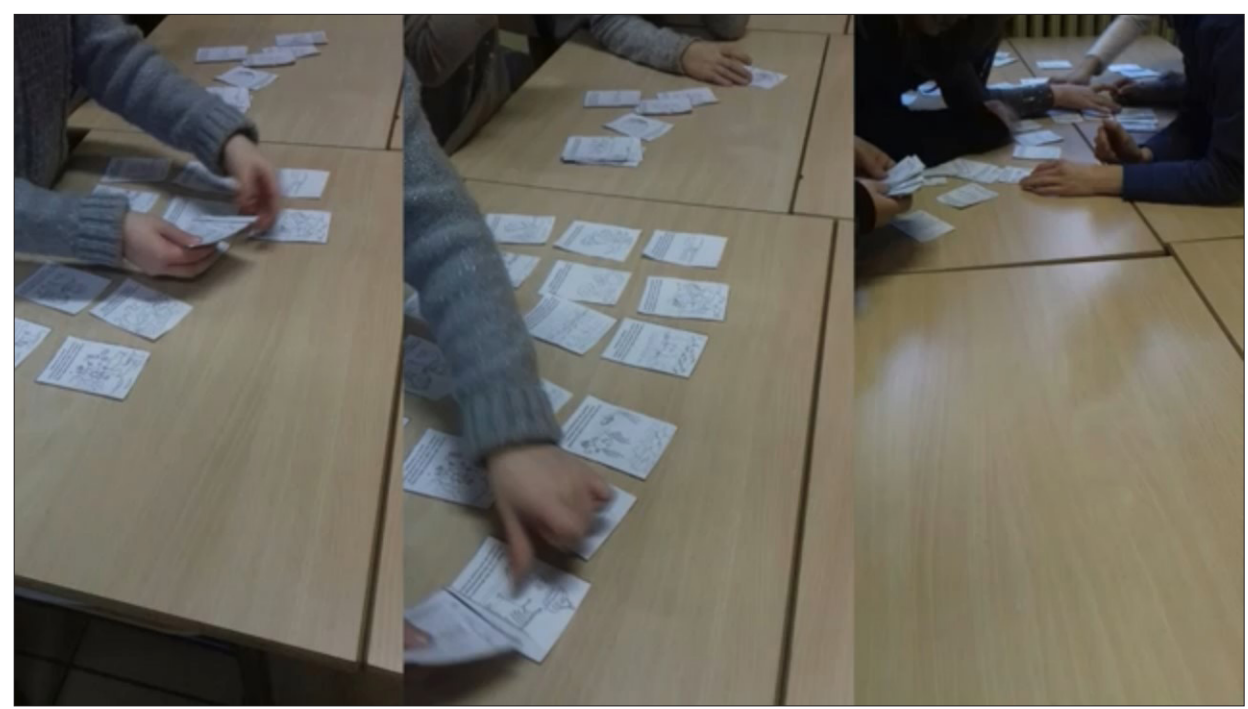

Le varie fasi del gioco del Memory sulla preistoria

intende così portarli a tematizzare in modo complesso, ossia a includere nel loro orizzonte concettuale delle pertinenze spaziali e temporali, di soggetto e d'azione. Ogni storia include più tipologie di pertinenze: in una sequenza di 4 carte 2 di queste si abbinano fra loro grazie a un'analogia di tipo spaziale (ad esempio nella sequenza relativa al raffreddamento della crosta terrestre, dove si propone una prospettiva geografica globale, oppure nella sequenza della deriva dei continenti, dove si parla esplicitamente di continenti), un'altra carta si aggrega al gruppo grazie a una pertinenza temporale, un'altra ancora per via della ricorrenza dello stesso soggetto. In ogni sequenza di carte è presente poi una pertinenza a esse trasversale, che le accomuna tutte: quella dell'azione. Le 


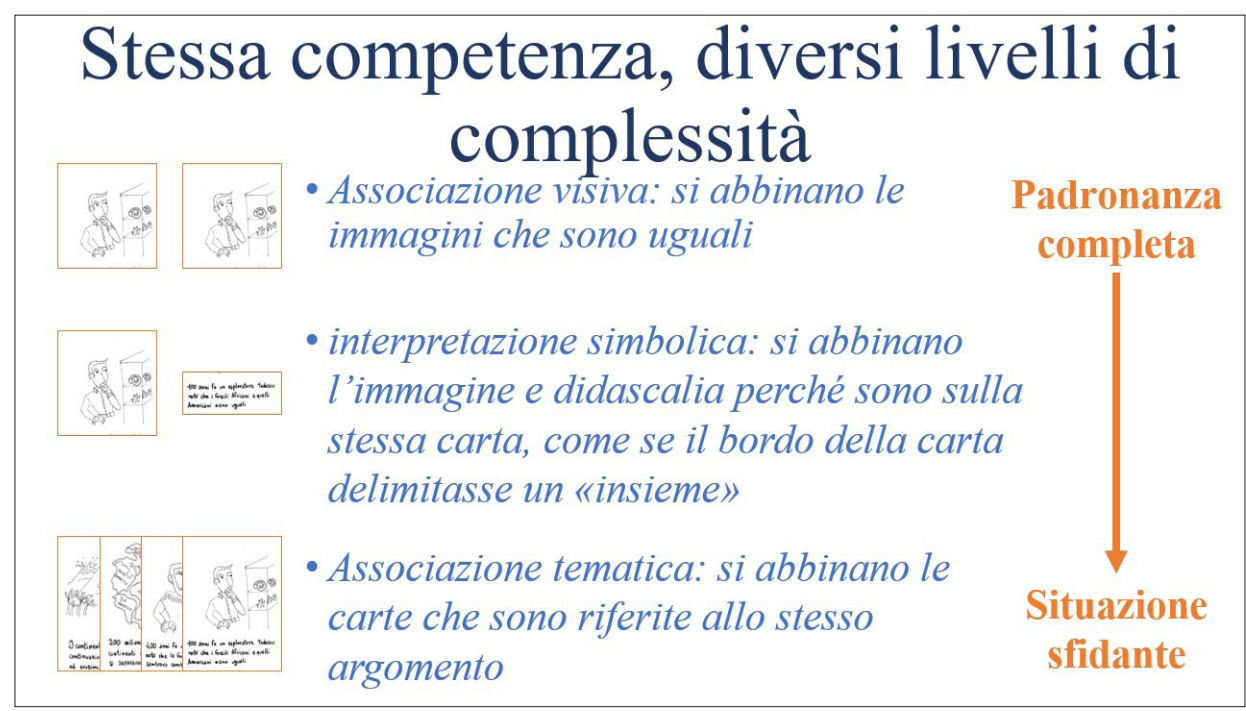

carte di ogni sequenza rappresentano quindi le varie fasi con le quali si svolge un'azione, o meglio, un processo, ad esempio il raffreddamento della crosta terrestre. Così non rappresentiamo la storia di un soggetto, o di un luogo, ma le fasi di un processo storico e in questo modo si stimola nei bambini una prospettiva storica di tipo 'processuale', che pone al centro della tematizzazione non i soggetti, ma di processi storici in cui questi sono inseriti.

\section{Un ragionamento personale, ma guidato}

Per esercitare una funzione di controllo sull'efficacia di questi processi di tematizzazione ed evitare che le sequenze vengano ricomposte in modo casuale, e non secondo l'individuazione di analogie tematiche, sono stati inseriti degli elementi di disturbo che introducono delle ambiguità tematiche e degli elementi di rottura, come nel caso della sequenza dell'era glaciale. In questo gruppo di carte, inseguendo una pertinenza soggettiva, i bambini si aspettano di trovare una quarta carta rappresentante il mammut, ma questa volutamente non esiste. In altre sequenze si è fatto leva invece sulla ricorsività dei soggetti per aiutare 
i bambini a intuire la relazione fra argomenti per loro completamente nuovi. Sempre in riferimento alla sequenza dell'era glaciale, è proprio la figura del mammut che agevola questo processo ed è stata inserita perché generalmente si propone il gioco prima che i bambini ne abbiano affrontato lo studio.

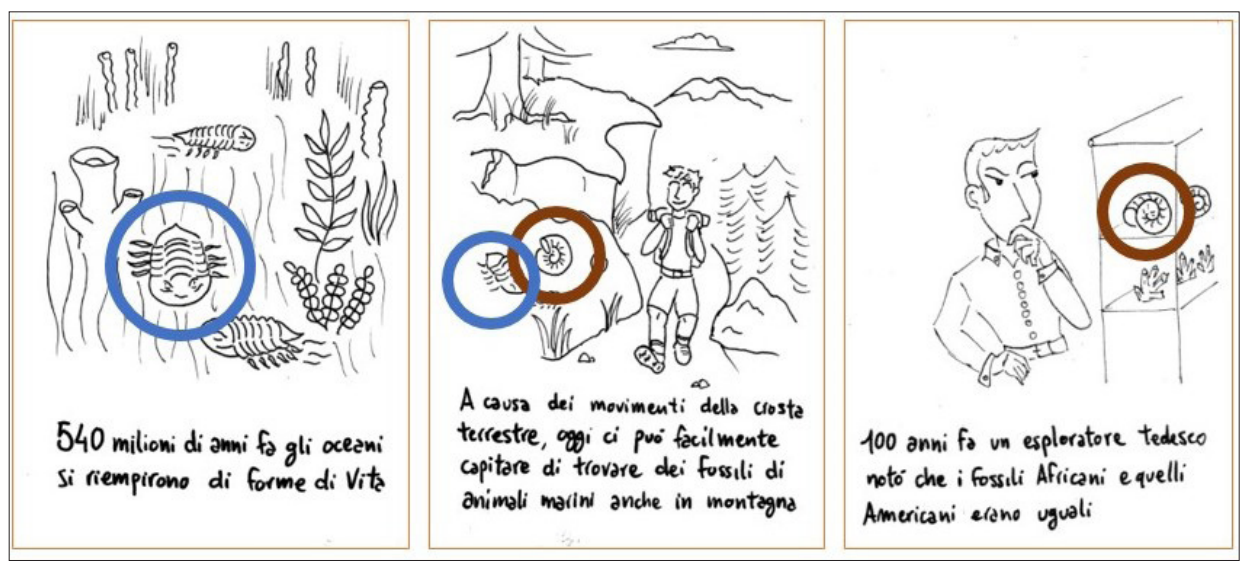

Elementi di disturbo presenti in carte appartenenti a sequenze diverse

Al termine del lavoro, quando tutte le carte sono state riordinate, il docente attacca alla lavagna le varie sequenze con del nastro adesivo, chiedendo ai bambini di dare un titolo a ciascuna di esse. La verbalizzazione è il coronamento del processo di esplicitazione di un tema: mentre giocavano essi stavano individuando e delimitando dei temi in

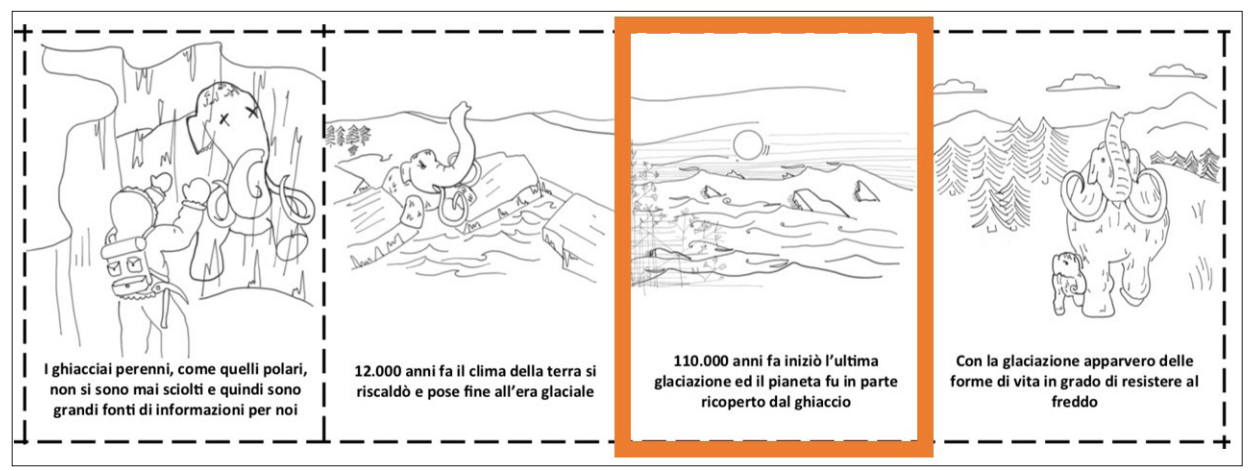

L'elemento mancante: i bambini si aspettano di trovare ancora una carta con la figura del mammut, e invece sono costretti a dover rintracciare la carta giusta basandosi su altri criteri rispetto a quello della ricorsività visiva. 
modo inconsapevole. Ora bisogna render visibile questo ragionamento in modo che i bambini sappiano ripercorrerlo razionalmente e coscientemente. Quando viene chiesto agli alunni di dare un titolo a ciascuna sequenza, essi sono portati ad analizzare i loro stessi procedimenti cognitivi ponendosi implicitamente una questione meta-cognitiva: «quali erano i tratti di similarità che mi hanno portato ad associare proprio queste carte?» Facendo emergere questi ragionamenti i bambini ne acquisiscono consapevolezza, possono identificare i propri procedimenti operativi e iniziano a sentire di essere in grado di capire la storia ed il senso del suo insegnamento. Iniziano a sentirsi capaci ed abili, e questo è molto importante perché tutti noi ci sentiamo molto più a nostro agio quando svolgiamo un'attività per la quale sappiamo di esser preparati. Il Memory storico è una delle attività più riuscite del percorso 'Giochi \& Civiltà', perché con un esiguo dispendio di tempo e di materiale (24 fotocopie per l'intera classe) consente di stimolare in modo mirato una delle competenze storiche più difficili da padroneggiare, approfondendo al contempo delle tematiche estremamente rilevanti e fornendo su di esse delle prospettive storico-didattiche aggiornate. Durante questa attività $\mathrm{i}$ bambini non si esercitano in una tematizzazione fine a sé stessa

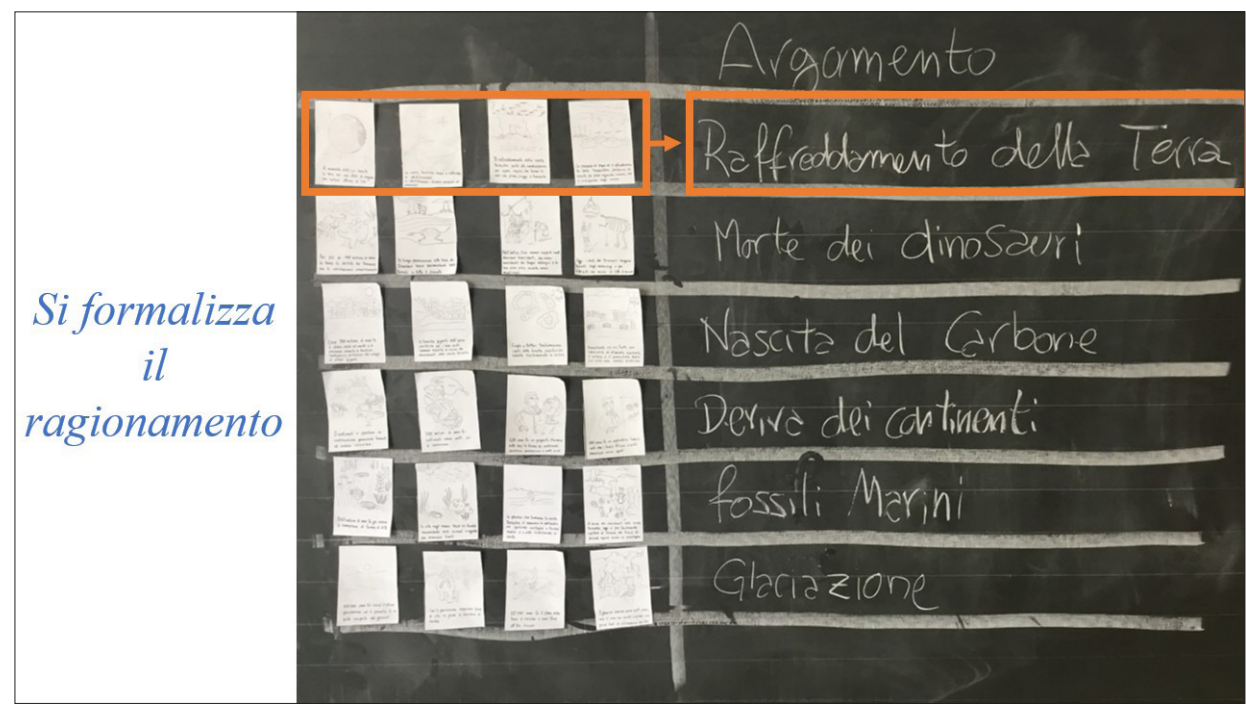

La lavagna al termine dell'attività 
ma operano con alcune delle conoscenze fondamentali del curricolo di storia, conoscenze irrinunciabili al fine di trasformare lo studio dell'epoca preistorica in un'occasione di formazione personale. Attraverso un gioco mostriamo ai bambini quanto possa essere importante il rapporto fra l'uomo e l'ambiente, fra l'uomo e le tracce storiche, e soprattutto fra il presente ed il passato. Le 6 sequenze che vanno ricomposte sono relative al raffreddamento della crosta terrestre, all'evoluzione della crosta terrestre, all'importanza della questione climatica, alla raccolta e conservazione delle tracce del passato. Sulle carte troviamo così rappresentato un cartografo incuriosito dalla forma dei continenti, un geologo che cerca di inferire a ritroso per capire come mai i fossili africani siano uguali a quelli dell'Almerica del sud, un camion che porta il carbone in una centrale elettrica odierna, un esploratore che guarda con stupore $\mathrm{i}$ resti di un mammut e un paleontologo che ricompone lo scheletro di un dinosauro in un museo. Quei tempi così remoti vengono quindi collegati al nostro presente attraverso un'attività che veicola una visione processuale della storia e mostra quanto il loro studio possa incuriosire, divertire, e formare la nostra visione del mondo.

\section{Esperienze condotte nelle scuole}

Nella fase di pre-test del progetto 'Giochi \& Civiltà', durata dal 2013 al 2018, questa attività è stata svolta in decine di classi III e in contesti molto differenti: dalla scuola di città a quella di paese, dalla classe senza alunni con BES alla classe composta al $90 \%$ da alunni stranieri con difficoltà linguistiche. Tutte le volte gli alunni hanno partecipato con entusiasmo e proattività. Nella fase di pre-test abbiamo spesso registrato $i$ tempi di risoluzione dell'esercizio da parte degli alunni, confrontandoli con quelli dei loro docenti e scoprendo che spesso i bambini riuscivano a ricomporre le 6 sequenze in modo più rapido, impiegando dai 30 ai 45 minuti. Nel 2018 l'attività è stata svolta nella scuola primaria di 
Zingonia, un istituto comprensivo noto per essere la scuola più multietnica della provincia di Bergamo, con alunni provenienti dall'Africa, America Latina, Europa orientale, vicino oriente e sub-continente indiano. Una classe composta quasi interamente da alunni di provenienza internazionale ha svolto l'attività in poco più di 20 minuti. Qualitativamente fu un dato sbalorditivo, poiché era la metà del tempo che eravamo abituati a registrare. Sarebbe interessante indagare le ragioni del risultato raggiunto dai bambini di Zingonia per capire se sia stato frutto di una maggiore flessibilità nella formulazione tematica dovuta all'interazione quotidiana fra persone con diversi background culturali e strutture mentali, oppure se sia stato determinato dall'approccio didattico fortemente esperienziale ed operativo adottato in quell'sstituto. Alcune settimane dopo, il 19 marzo 2018, durante un corso di formazione docenti, la stessa attività è stata riproposta a una sessantina di insegnanti dell'stituto Comprensivo di Corinaldo, i quali, pur lavorando in gruppi da 5-6 docenti ciascuno, non hanno potuto portare a termine l'attività nell'arco di 40 minuti. Questo fatto ci dà l'idea delle potenzialità cognitive degli alunni della scuola primaria che l'attuale metodologia di insegnamento della storia lascia inespresse.

\section{Il progetto ‘Giochi \& Civiltà'}

Il progetto 'Giochi \& Civiltà’ è composto da 15 attività distribuite fra la classe III e V della scuola primaria. La loro sequenza è stata strutturata per poter agevolare la sedimentazione di quelle pratiche operative che portano gli alunni a comprendere la storia, a capire che la finalità del suo insegnamento non è la memorizzazione di contenuti e informazioni puntuali, ma un modo di guardare il mondo. Così, l'attività del Memory acquista un maggiore senso se pensiamo che è inserita in un ciclo di 5 lezioni che, distribuite su tutto l'arco dell'anno scolastico, portano 
gli alunni a confrontarsi in modi sempre nuovi con la tematizzazione e con le conoscenze imprescindibili relative alla preistoria.

Una sperimentazione monitorata del progetto 'Giochi \& Civiltà' era stata iniziata nell'anno scolastico in 2019-2020, con lo scopo di raccogliere ulteriori risultati, ma è stata interrotta dall'insorgenza della pandemia di Coronavirus. Attualmente, non possiamo far altro che riportare i commenti di alcuni alunni della Scuola Primaria Locatelli di Bergamo risalenti all'anno scolastico 2017-2018. Dopo 3 anni di test svolti in parallelo su 3 gruppi classe che hanno seguito il progetto 'Giochi \& Civiltà' dalla III alla V, ho chiesto agli alunni di scrivermi un piccolo commento anonimo su dei post-it. Ecco due risposte che potrebbero far capire le potenzialità dell'attività ludica applicata alla didattica disciplinare e che mostrano la capacità dei bambini sia di sviluppare una prospettiva meta-cognitiva sia di migliorare il loro rapporto con la disciplina:

"Marco il primo anno io pensavo che tu fossi solo un semplice insegnante di storia, ma invece avevo capito che tu ci vuoi far "ingrandire il cervello" di storia. Per questo ti ringrazio al 1000x1000.»

«Sono veramente felice di questi tre anni passati assieme a te e penso che sia stata un'esperienza eccezionale: da grande vorrei forse fare il tuo lavoro.»

Anche se non abbiamo avuto modo di testare in modo ufficiale il percorso 'Giochi \& Civiltà' nelle scuole, le risposte ottenute ci fanno ben sperare: sembra che i bambini che hanno studiato la storia in questo modo, giocando, abbiano capito come usare le informazioni storiche per ricavarne delle conoscenze.

\section{Bibliografia:}

Mattozzi, Ivo. 1990. "Morfologia della conoscenza storiografica e didattica." In La cultura storica: un modello di costruzione, a cura di Ivo Mattozzi, 3-32. Faenza: Faenza editrice. 
Mattozzi, Ivo. 1992. "Che il piccolo storico sia." I viaggi di Erodoto (16): 168-180.

Mattozzi, Ivo. 2005. "Un modello curricolare di educazione storica di base". In Un curricolo per la Storia, a cura di Ivo Mattozzi, 13-62. Bologna: Cappelli Editore.

Mattozzi, Ivo. 2011. Pensare la Storia da insegnare, Vol.1., Castel San Guelfo di Bologna: Cenacchi Editrice.

Tibaldini, Marco, Scacchi, Mattia. 2017. "Facciamo finta che... imparare con la fantasia." In La storia oltre i manuali. Come usare testi storiografici e testi di finzione storica, a cura di Dalola, Daniela, Rabitti Maria Teresa, 171-182. Padova: Mnamon. 